\title{
RELATIONS BETWEEN RANKS OF MATRIX POLYNOMIALS
}

\section{VASILE POP}

Technical University of Cluj-Napoca

Str. C. Daicoviciu 15

400020, Cluj-Napoca

Romania

e-mail: vasile.pop@math.utcluj.ro

\begin{abstract}
We show that the sum of the ranks of two matrix polynomials is the same as the sum of the rank of the matrix obtained by applying the greatest common divisor of the polynomials, with the rank of the matrix obtained by applying the least common multiple of the polynomials. Many applications, for older or more recent problems, of this result are obtained.
\end{abstract}

2020 Mathematics Subject Classification: 15A24.

Keywords and phrases: rank, matrix, polynomial.

Received February 05, 2021; Revised March 25, 2021

(C) 2021 Scientific Advances Publishers

This work is licensed under the Creative Commons Attribution International License (CC BY 3.0).

http://creativecommons.org/licenses/by/3.0/deed.en_US

Open Access (cc) (1)




\section{Introduction}

Let $(K,+, \cdot)$ be a field, $K[X]$ be the ring of polynomials with coefficients in $K$ and $\mathscr{N}_{n}(K)$ be the ring of square matrices of order $n \geq 2$ with coefficients in $K$. Let $d:=(f, g)$ denote the greatest common divisor and $m:=[f, g]$ denote the lowest common multiple of the polynomials $f, g$. The main result of this paper is the following theorem.

Theorem 1 (Sum of ranks of matrix polynomials). For any two

polynomials $f, g \in K[X]$ and for any matrix $A \in \mathscr{N}_{n}(K)$ the following relation holds:

$$
\operatorname{rank} f(A)+\operatorname{rank} g(A)=\operatorname{rank} d(A)+\operatorname{rank} m(A) .
$$

From the above relation one can obtain other interesting relations, particular theorems that characterize some matrices as idempotent, tripotent, involutive matrices, and other results which may be well known for experts. These are exposed in Section 3 where we reviewed some particular results obtained in [1], [2], and [3].

\section{Proof of Theorem 1}

We will prove Theorem 1 using the method of elementary transformations in block-partitioned matrices, which appears in [5, Chapter]. We choose this reference on matrix theory because it is suitable for the applications we have in mind in Section 3 with respect different original problems and results which are proposed in [1], [2] and [3].

The main result that we use is the following well-known lemma, which is true since $K[X]$ is an Euclidian domain.

Lemma 1 ([4, Theorem 3.31]). If $f, g \in K[X]$, then there are $\varphi_{1}, \varphi_{2} \in K[X]$ such that

$$
f \cdot \varphi_{1}+\varphi_{2} \cdot g=d
$$


Proof (of Theorem 1). For the matrix $A$ the relation (1) becomes

$$
f(A) \cdot \varphi_{1}(A)+\varphi_{2}(A) \cdot g(A)=d(A) .
$$

We start with the block matrix $B \in \mathscr{N}_{2 n}(K)$

$$
B=\left[\begin{array}{c|c}
f(A) & 0 \\
\hline 0 & g(A)
\end{array}\right]
$$

with $\operatorname{rank} B=\operatorname{rank} f(A)+\operatorname{rank} g(A)$ and we perform the following elementary transformations:

$$
\begin{gathered}
{\left[\begin{array}{c|c}
f(A) & 0 \\
\hline 0 & g(A)
\end{array}\right] \stackrel{C_{1}}{\longrightarrow}\left[\begin{array}{c|c}
f(A) & f(A) \cdot \varphi_{1}(A) \\
\hline 0 & g(A)
\end{array}\right] \stackrel{L_{1}}{\longrightarrow}} \\
{\left[\begin{array}{c|c}
f(A) & d(A) \\
\hline 0 & g(A)
\end{array}\right] \stackrel{L_{2}}{\longrightarrow}\left[\begin{array}{c|c}
f(A) & d(A) \\
\hline-m(A) & 0
\end{array}\right] \stackrel{C_{2}}{\longrightarrow}} \\
{\left[\begin{array}{c|c}
0 & d(A) \\
\hline-m(A) & 0
\end{array}\right]=C,}
\end{gathered}
$$

so that $\operatorname{rank} C=\operatorname{rank} d(A)+\operatorname{rank} m(A)$.

The matrices used for the elementary transformations on columns $\left(C_{1}, C_{2}\right)$, and on rows $\left(L_{1}, L_{2}\right)$ are

$$
\begin{gathered}
C_{1}=\left[\begin{array}{c|c}
I_{n} & \varphi_{1}(A) \\
\hline 0 & I_{n}
\end{array}\right], \quad L_{1}=\left[\begin{array}{c|c}
I_{n} & \varphi_{2}(A) \\
\hline 0 & I_{n}
\end{array}\right], \\
C_{2}=\left[\begin{array}{c|c}
I_{n} & 0 \\
\hline-\psi_{2}(A) & I_{n}
\end{array}\right], \quad L_{2}=\left[\begin{array}{c|c}
I_{n} & 0 \\
\hline-\psi_{1}(A) & I_{n}
\end{array}\right],
\end{gathered}
$$


where $g(A)=\psi_{1}(A) \cdot d(A)$ and $f(A)=d(A) \cdot \psi_{2}(A)$, with $\psi_{1}$ and $\psi_{2}$ being the polynomials obtained after dividing $f$ and $g$, respectively, by $d$. The relation (2) is used in the second step, and the relation $m=\psi_{1} f$ is used in the third one. Note that this last relation is a consequence of the identities $m d=f_{g}$ and $g=\psi_{1} d$.

Since $C=L_{2} \cdot L_{1} \cdot B \cdot C_{1} \cdot C_{2}$ and $C_{1}, C_{2}, L_{1}, L_{2}$ are invertible, it follows that $\operatorname{rank} B=\operatorname{rank} C$, and this concludes the proof.

\section{Corollaries}

Notice that Theorem 1 is trivially true if we have the same $f=g$, but if we want to obtain new problems or interesting corollaries we apply our Theorem 1 only when $f$ and $g$ are two distinct polynomials.

Corollary 1. If $f$ and $g$ are coprime polynomials, then for any matrix $A \in \mathscr{C}_{n}(K)$ the following relation holds:

$$
\operatorname{rank} f(A) \cdot g(A)+n=\operatorname{rank} f(A)+\operatorname{rank} g(A) .
$$

The following corollary appears in [3].

Corollary 2. If $f$ and $g$ are coprime polynomials, then $f(A) \cdot g(A)=0$ if and only if $f(A)+\operatorname{rank} g(A)=n$.

Corollary 3 ([1], [2]). The matrix $A \in \mathscr{N}_{n}(K)$ is idempotent $\left(A^{2}=A\right)$ if and only if

$$
\operatorname{rank} A+\operatorname{rank}\left(I_{n}-A\right)=n
$$

Proof. Take the polynomials $f(x)=x, g(x)=1-x$ and then we have $d(x)=1, m(x)=x-x^{2}$. Now we apply Corollary 2. 
Corollary 4 ([1], [2]). Let $K$ be a field of characteristic different than 2. The matrix $A \in \mathscr{N}_{n}(K)$ is involutive $\left(A^{2}=I_{n}\right)$ if and only if

$$
\operatorname{rank}\left(I_{n}-A\right)+\operatorname{rank}\left(I_{n}+A\right)=n .
$$

Proof. We apply Corollary 2 for the polynomials $f(x)=1-x$, $g(x)=1+x$.

Corollary 5 ([2]). If $K$ is a field with characteristic different than 2, then the following statements are equivalent:

(1) The matrix $A$ is tripotent $\left(A^{3}=A\right)$.

(2) $\operatorname{rank} A+\operatorname{rank}\left(I_{n}-A^{2}\right)=n$.

(3) $\operatorname{rank}\left(I_{n}-A\right)+\operatorname{rank}\left(A+A^{2}\right)=n$.

(4) $\operatorname{rank} A+\operatorname{rank}\left(I_{n}-A\right)+\operatorname{rank}\left(I_{n}+A\right)=2 n$.

Proof. " $(1) \Leftrightarrow(2)$ ". We apply Corollary 2 for the polynomials $f(x)=x, \quad g(x)=1-x^{2}$.

“(1) $\Leftrightarrow(3)$ ”. We apply Corollary 2 for the polynomials $f(x)=1-x$, $g(x)=x+x^{2}$.

“(2) $\Leftrightarrow(4)$ ". It is enough to prove the relation

$$
n+\operatorname{rank}\left(I_{n}-A^{2}\right)=\operatorname{rank}\left(I_{n}-A\right)+\operatorname{rank}\left(I_{n}+A\right),
$$

which is true by Corollary 1 , for $f(x)=1-x$ and $g(x)=1+x$.

Corollary 6. Let $A \in \mathscr{N}_{n}(K)$ and $f_{A} \in K[X]$ be its characteristic polynomial, which we decompose in a product of polynomials that are pairwise coprime

$$
f_{A}=f_{1} \cdot f_{2} \cdots f_{k}
$$


Then we have the relation

$$
\operatorname{rank} f_{1}(A)+\operatorname{rank} f_{2}(A)+\cdots+\operatorname{rank} f_{k}(A)=(k-1) n .
$$

Proof. We apply Corollary 2 for $f=f_{1} \cdot f_{2} \cdots f_{k-1}, g=f_{k}$ and obtain

$$
\operatorname{rank} f(A)+\operatorname{rank} f_{k}(A)=n \text {. }
$$

We apply now Corollary 1 for $f=f_{1} \cdot f_{2} \cdots f_{k-2}, g=f_{k-1}$ and obtain

$$
\operatorname{rank}\left(f_{1} \cdot f_{2} \cdots f_{k-2}\right)(A)+\operatorname{rank} f_{k-1}(A)+\operatorname{rank} f_{k}(A)=2 n .
$$

By induction, we obtain the result.

Corollary 7. Let $K$ be a field of characteristic different than 2. For any matrix $A \in \mathscr{N}_{n}(K)$ the following relation is verified:

$$
\operatorname{rank}\left(A+A^{2}\right)+\operatorname{rank}\left(A-A^{2}\right)=\operatorname{rank} A+\operatorname{rank}\left(A-A^{3}\right) .
$$

Proof. We apply Theorem 1 with

$$
f(x)=x+x^{2}, \quad g(x)=x-x^{2}, \quad d(x)=x, \quad m(x)=x-x^{3} .
$$

The last corollary is easily obtained by applying Theorem 1 to suitable polynomials. We can recover the results which were published in [2] and various problems, proposed by the author to some mathematical competitions for students.

Corollary 8 ([2]). Let $K$ be a field of characteristic different than 2. For any matrix $A \in \mathscr{N}_{n}(K)$, the following statements are equivalent:

(1) $A^{3}=A^{5}$.

(2) $\operatorname{rank} A^{3}+\operatorname{rank}\left(I_{n}-A^{2}\right)=n$.

(3) $\operatorname{rank}\left(I_{n}-A\right)+\operatorname{rank}\left(A^{3}+A^{4}\right)=n$.

(4) $\operatorname{rank}\left(I_{n}+A\right)+\operatorname{rank}\left(A^{3}-A^{4}\right)=n$. 
(5) $\operatorname{rank} A^{3}+\operatorname{rank}\left(I_{n}-A\right)+\operatorname{rank}\left(I_{n}+A\right)=2 n$.

(6) $\operatorname{rank}\left(A-A^{2}\right)+\operatorname{rank}\left(A^{3}+A^{4}\right)=\operatorname{rank} A$.

(7) $\operatorname{rank}\left(A+A^{2}\right)+\operatorname{rank}\left(A^{3}-A^{4}\right)=\operatorname{rank} A$.

(8) $\operatorname{rank}\left(A^{3}+A^{4}\right)+\operatorname{rank}\left(A^{3}-A^{4}\right)=\operatorname{rank} A^{3}$.

\section{Acknowledgements}

We are grateful to an unknown referee for his remarks which improved the first version of this paper.

\section{References}

[1] V. Pop, The characterization of same linear maps using the rank, Journal of Science and Arts 3(36) (2016), 225-228.

[2] V. Pop, The method of elementary operations in block matrices for the determination of the annihilator polynomials of some matrices, Journal of Science and Arts 4(37) (2016), 295-302.

[3] V. Pop, The decomposition of some annihilator polynomials of linear maps in coprime polynomials, Journal of Science and Arts 4(41) (2017), 647-650.

[4] J. J. Rotman, Advanced Modern Algebra, Prentice Hall (Second Printing), 2003

[5] F. Zhang, Matrix Theory: Basic Results and Techniques, Springer, New York, 1999. 